\title{
Incidence, treatment, and survival analysis in esophageal neuroendocrine carcinoma population
}

\author{
Zhenhua Li ${ }^{1 \#}$, Jiali Hü ${ }^{2 \#}$, Pifeng Chen ${ }^{3}$, Zhi Zeng ${ }^{4}$ \\ ${ }^{1}$ Department of Cardiothoracic Surgery, Xianning Central Hospital, The First Affiliated Hospital of Hubei University of Science and Technology, \\ Xianning, China; ${ }^{2}$ Department of Pathology, Jiujiang University Clinic College/Hospital, Jiujiang, China; ${ }^{3}$ Department of Pediatric Surgery, Jiujiang \\ Maternal and Child Health Hospital, Jiujiang, China; ${ }^{4}$ Department of Pathology, Xianning Central Hospital, The First Affiliated Hospital of Hubei \\ University of Science and Technology, Xianning, China \\ Contributions: (I) Conception and design: Z Zeng, Z Li; (II) Administrative support: Z Zeng; (III) Provision of study materials or patients: Z Zeng, Z \\ Li; (IV) Collection and assembly of data: J Hu, Z Li; (V) Data analysis and interpretation: Z Zeng, P Chen; (VI) Manuscript writing: All authors; (VII) \\ Final approval of manuscript: All authors. \\ \#These authors contributed equally to this work. \\ Correspondence to: Zhi Zeng. Department of Pathology, Xianning Central Hospital, The First Affiliated Hospital of Hubei University of Science and \\ Technology, 228 Jingui Road, Xianan District, Xianning, Hubei, China. Email: path080706@163.com.
}

Background: As a subtype of esophageal neuroendocrine neoplasms (ENENs), esophageal neuroendocrine carcinoma (ENEC) is rare. ENEC can be poorly differentiated and highly aggressive. This study aims to illustrate the incidence, treatment, and prognosis of ENEC by using a population-based database.

Methods: We collected clinicopathological data [1975-2016] of ENEC, esophageal adenocarcinoma (EACA), and esophageal squamous cell carcinoma (ESqCC) from the Surveillance, Epidemiology and End Results (SEER) database, and analyzed their incidence, treatment, and prognosis.

Results: A total of 60,238 cases were in our study cohort, including ENEC ( $\mathrm{n}=686)$, EACA ( $\mathrm{n}=26,575)$, and $\mathrm{ESqCC}(\mathrm{n}=32,977)$. The incidence of ENEC in 2016 was 0.044 per 100,000 persons. The tumor grade of ENEC was significantly higher than that of EACA and ESqCC $(\mathrm{P}<0.001)$. Both cancer-specific survival (CSS) and overall survival (OS) of ENEC were significantly worse than those of EACA and ESqCC $(\mathrm{P}<0.001)$. Kaplan-Meier analysis showed significant improvement of CSS and OS by surgery, radiotherapy, and chemotherapy (all $\mathrm{P}<0.001$ ). Besides, compared with monotherapies, combinational therapies brought more benefits to both CSS and OS of patients with ENEC.

Conclusions: Our population-based evidence revealed that ENEC, as a rare cancer, had a worse prognosis, compared with EACA and ESqCC. Surgery, chemotherapy, and radiotherapy can all improve the prognosis of ENEC patients. Combinational therapy can yield a better prognosis than monotherapy.

Keywords: Esophageal neoplasms; carcinoma, neuroendocrine; survival analysis

Submitted Nov 29, 2019. Accepted for publication May 29, 2020.

doi: $10.21037 /$ tcr-19-2650

View this article at: http://dx.doi.org/10.21037/tcr-19-2650

\section{Introduction}

Esophageal neuroendocrine neoplasms (ENENs) are rare (1). According to World Health Organization classification, ENENs have three categories: lowgrade (G1) neuroendocrine tumor, intermediate-grade (G2) neuroendocrine tumor, and high-grade (G3) neuroendocrine carcinoma (NEC) (2). Esophageal neuroendocrine carcinoma (ENEC) is the most poorly differentiated subtype of ENENs. ENEC mainly includes small cell and large cell variants. Microscopically, small celltype ENEC has small cancer cells in similar sizes arranged in a row with unclear boundaries, and the nucleus is in round/short/spindle-like shape (3). Large cell-type ENEC 
has tumor cells with more than three-fold size as that of lymphocytes arranged in sheets/nests and less cytoplasm, and the nucleus is in round/oval/spindle-like shape (4). For imaging exams, in addition to ultrasound, computed tomography (CT), and magnetic resonance imaging, pathological slices dealt with immunohistochemistry (e.g., $\mathrm{Ki}-67$ staining) can provide a precise diagnosis of ENEC $(5,6)$.

Compared with other commonly seen esophageal cancers like esophageal adenocarcinoma (EACA) and esophageal squamous cell carcinoma (ESqCC), ENEC has more aggressive biological behavior $(7,8)$. The lesions of ENEC are mainly found in the middle and lower parts of the esophagus, probably because of the unequal distribution of endocrine cells in the esophagus (9). Principal symptoms of ENEC include dysphagia, gastroesophageal reflux, chest pain, and weight loss, which can also appear in other esophageal cancers $(10,11)$. However, because of ENEC's endocrinal property, patients may also manifest different hormone-related syndromes $(8,12)$.

Treatments of ENEC have not been well studied. Besides, there is a lack of population-based analysis on ENEC due to its rarity. In this study, we examined the Surveillance, Epidemiology, and End Results (SEER) registry to evaluate the incidence, treatment, and prognosis of ENEC.SEER database is a powerful tool to get information about rare cancers. We analyzed the clinicopathological characteristics of the ENEC population. We also compared the different prognosis of treatments, including surgery, chemotherapy, and radiotherapy. As such, this study can help improve the understanding of ENEC.

We present the following article in accordance with the STROBE reporting checklist (available at http://dx.doi. org/10.21037/tcr-19-2650).

\section{Methods}

\section{Patient and tumor characteristics}

We extracted information about ENEC, ESqCC, and EACA from 1975 to 2016 from the SEER 18 database. Subsequently, we analyzed the incidence, frequency, and survival data. Due to omitted patient identifiers, this study did not have to get the approval of Institutional Review Board.

We used behavior code 3 to identify malignant tumors. After the filtration, only cases identified by site-specific codes of the esophagus (C15.0 - C15.5, C15.8 and C15.9) were included, based on the International Classification of Disease for Oncology, 3rd Edition (ICD-O-3). Histology/ behavior code representing different subtypes of NEC included typical carcinoid (8240/3), atypical carcinoid (8249/3), large cell NEC (8013/3), small cell NEC (8041/3), and NEC NOS (8246/3). Histology/behavior code of ESqCC and EACA was 8140/3 and 8070/3, respectively. Tumors with diagnostic confirmation (microscopically/ not microscopically confirmed) were included for further analysis. Tumors that are not malignant, not first occurring, and without histology/exfoliative cytology/microscopic/ laboratory test or marker confirmed were excluded from the initial cohort.

Classification and statistical analysis were executed based on clinicopathological parameters, including sex, ethnicity, marital status, age, tumor location, and grade. Tumor features included Tumor-Node-Metastasis (TNM) stage, American Joint Committee on Cancer (AJCC) staging, SEER histological stage, and intervention type. Rightcensored data of the overall survival (OS) and cancerspecific survival (CSS) analysis included cases that survived the deadline, lost follow-up, or died of other reasons.

\section{Incidence and survival}

All rates were reported per 100,000 persons. The age of the patients was adjusted to the 2000 US Standard Population (19 age groups, census P25 - 1130) standard. The annual percent change was demonstrated in incidence using 1-year endpoints to analyze the survival rate. OS and CSS were used to reflect survival trends and build the Kaplan-Meier model.

\section{Statistical analysis}

After the extraction of frequency, incidence, and survival data in all cases from the SEER 18 database, statistical analysis was executed in SPSS software (version 24.0, SPSS Inc., Chicago, IL, USA) and SEER*Stat 8.3.6 software (National Cancer Institute, Bethesda, Maryland, USA). Data of incidence were analyzed by weighted least squares to generate annual percentage change based on 1-year endpoints in SEER*Stat 8.3.6 software. The student's $t$-test and Chi-square test were respectively used to deal with continuous and categorical variables. $\mathrm{P}$ values were twotailed, and those less than 0.05 was considered as significant. 


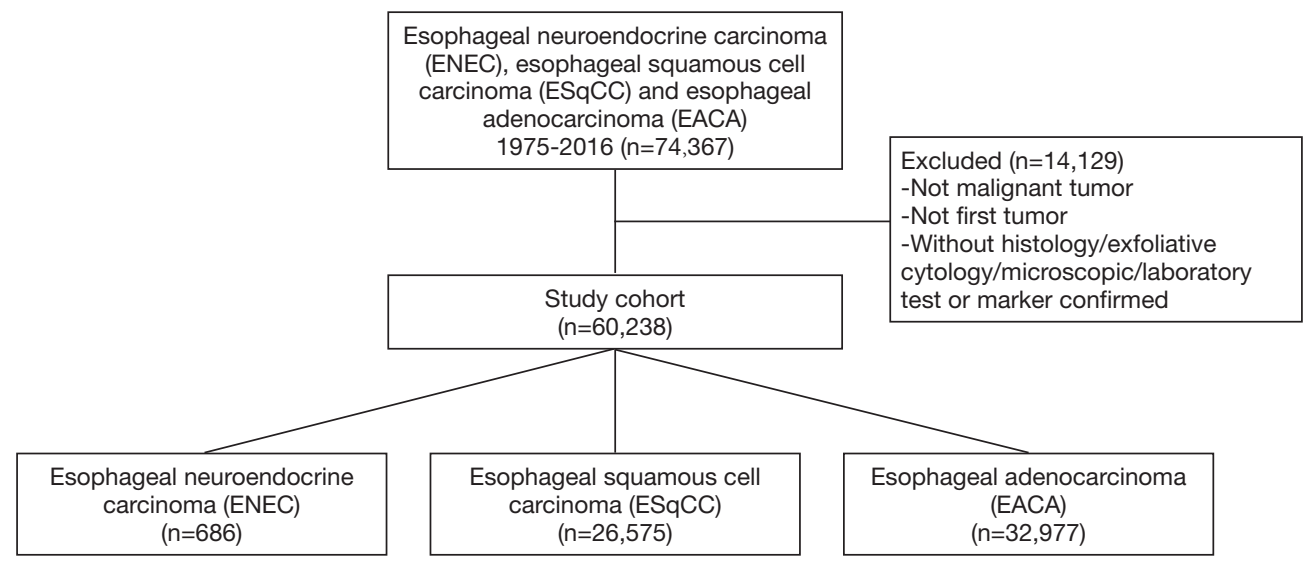

Figure 1 Workflow chart of selection of patients with ENEC, ESqCC, or EACA from the SEER database. ENEC, esophageal neuroendocrine carcinoma; ESqCC, esophageal squamous cell carcinoma; EACA, esophageal adenocarcinoma.

\section{Results}

\section{Patient characteristics}

In the SEER 18 database, a total of 60,238 patients were identified with ENEC $(n=686), \operatorname{ESqCC}(n=26,575)$, or EACA $(n=32,977)$, during 1975 to 2016 after exclusion (Figure 1). All demographic characteristics were demonstrated in Table 1. Patients with ENEC were comparatively older (median: 67, mean: 67.05, $\mathrm{P}<0.001$ ) than patients with EACA (median: 65, mean: 65.30) and ESqCC (median: 67, mean: 66.59). Furthermore, ENEC (median: $5.7 \mathrm{~cm}$, mean: $6.1 \mathrm{~cm}, \mathrm{P}<0.001$ ) had significantly larger tumor size, compared with EACA (median: $4.5 \mathrm{~cm}$, mean: $4.9 \mathrm{~cm}$ ) and $\mathrm{ESqCC}$ (median: $5.0 \mathrm{~cm}$, mean: $5.4 \mathrm{~cm}$ ). Patients with ENEC had larger proportion of high-grade tumors (I: $1.2 \%$; II: 0.9\%; III: 37.2\%; IV: 30.2\%, P<0.001). Besides, compared with EACA and ESqCC, patients with ENEC had larger proportion receiving chemotherapy (Yes: $62.7 \%$, No: $37.3 \%$ ) and less proportion receiving radiotherapy (Yes: 43\%, No: 56.3\%).

\section{Tumor characteristics}

For ENEC, tumor-node-metastasis (TNM), American Joint Committee on Cancer (AJCC) stage, and SEER stage can be checked in Table 2. In ENEC, T1 stage was the most identified (38.6\%), followed by the stage of T3 $(29.1 \%)$, T4 $(22.8 \%)$, and T2 (9.4\%). Lymph node involvement included N0 (50\%), N1 (59.8\%), N2 (6.7\%), and N3 (2.4\%). For distant metastasis, metastasis (M1) appeared in $49 \%$ of cases, while $51 \%$ of cases were without distant metastasis.
For the AJCC stage, the largest proportion belonged to stage IV (57.4\%), followed by stage III (17.2\%), stage II (16.6\%), and stage I (8.9\%). Besides, the SEER stage revealed that tumors with distant metastasis accounted for the largest proportion (54.5\%) among all stages, including localized stage, regional metastasis, distant metastasis, and the unstaged.

\section{Incidence analysis}

The incidence of ENEC was verified to be 0.044 per 100,000 between 2000 and 2016, after age adjustment to the 2000 US Standard Population (19 age groups, census P25 - 1130) standard (Table 1). Although annual percentage change [2000-2016] was $1.272 \%$, such change of incidence made no significance $(\mathrm{P}=0.060 ;$ Table 1 , Figure 2). In contrast, the incidence of EACA and ESqCC was 2.257 and 1.055 per 100,000 persons, which exhibited a significant annual percentage change [2000-2016] of $0.584 \%(\mathrm{P}=0.005)$ and $-2.877 \%(\mathrm{P}<0.001)$, respectively (Table 1, Figure 2).

\section{Treatment and survival analysis}

The profile of treatments among patients with ENEC was summarized in Table 3. In detail, surgery, chemotherapy, and radiotherapy all showed protective effect on ENEC patients (HR (95\% CI) by univariate analysis, surgery: 0.5 (0.38-0.666), $\mathrm{P}<0.001$; chemotherapy: 0.52 (0.45-0.62), $\mathrm{P}<0.001$; radiotherapy: 0.59 (0.50-0.69), $\mathrm{P}<0.001$, and HR (95\% CI) by multivariate analysis, surgery: 0.37 (0.19-0.74), P=0.005; chemotherapy: $0.53(0.35-0.82)$, 
Table 1 Patient clinical and pathological features of ENEC, EACA, and ESqCC

\begin{tabular}{|c|c|c|c|c|}
\hline Parameter, n (\%) & ENEC $(n=686)$ & EACA $(n=32,977)$ & ESqCC $(n=26,575)$ & $\mathrm{P}$ \\
\hline \multicolumn{5}{|l|}{ Year (\%) } \\
\hline $1975-1986$ & $58(8.5)$ & $1,177(3.6)$ & $5,645(21.2)$ & $<0.001$ \\
\hline 1987-1996 & 79 (11.5) & $3,194(9.7)$ & 4,833 (18.2) & \\
\hline $1997-2006$ & $209(30.5)$ & $11,100(33.7)$ & $7,851(29.5)$ & \\
\hline \multicolumn{5}{|l|}{ Age (years) } \\
\hline Median & 67 & 65 & 67 & $<0.001$ \\
\hline Mean & 67.05 & 65.30 & 66.59 & \\
\hline Range & $24-93$ & $19-101$ & $11-103$ & \\
\hline \multicolumn{5}{|l|}{ Size (cm) } \\
\hline Mean & 6.1 & 4.9 & 5.4 & \\
\hline Range & $0.1-25.0$ & $0.1-98.9$ & $0.1-98.9$ & \\
\hline \multicolumn{5}{|l|}{ Sex $(\%)$} \\
\hline Male & $464(67.6)$ & $28,262(85.7)$ & $17,661(66.5)$ & $<0.001$ \\
\hline Female & $222(32.4)$ & 4,715 (14.3) & 8,914 (33.5) & \\
\hline \multicolumn{5}{|l|}{ Race/ethnicity (\%) } \\
\hline White & $554(80.8)$ & $31,236(94.7)$ & $16,685(62.8)$ & $<0.001$ \\
\hline Black & 89 (13.0) & $852(2.6)$ & 7,352 (27.7) & \\
\hline II & $6(0.9)$ & $11,073(33.6)$ & 20,654 (34.3) & \\
\hline III & $255(37.2)$ & $13,990(42.4)$ & 23,967 (39.8) & \\
\hline IV & $207(30.2)$ & $443(1.3)$ & 978 (1.6) & \\
\hline Unknown & $210(30.6)$ & 5701 (17.3) & $11,400(18.9)$ & \\
\hline \multicolumn{5}{|l|}{ Surgery (\%) } \\
\hline Yes & $69(10.2)$ & $11,113(34.1)$ & $5,209(20.4)$ & $<0.001$ \\
\hline No & 607 (89.8) & 21,496 (65.9) & 20,385 (79.6) & \\
\hline \multicolumn{5}{|l|}{ Chemotherapy (\%) } \\
\hline Yes & $430(62.7)$ & $18,881(57.3)$ & $13,103(49.3)$ & $<0.001$ \\
\hline No & $256(37.3)$ & $14,096(42.7)$ & $13,472(50.7)$ & \\
\hline
\end{tabular}

Table 1 (Continued) 
Table 1 (Continued)

\begin{tabular}{lccc}
\hline Parameter, $n(\%)$ & ENEC $(n=686)$ & EACA $(n=32,977)$ & ESqCC $(n=26,575)$ \\
\hline Radiotherapy (\%) & $297(43.7)$ & & $P$ \\
Yes & $383(56.3)$ & $17,003(52.1)$ & $17,016(65.1)$ \\
No & 0.044 & $15,624(47.9)$ & $9,126(34.9)$ \\
Incidence [2016] & $1.272(P=0.060)$ & $0.584(P=0.005)$ & 1.055 \\
Annual percentage change & & & $-2.877(P<0.001)$ \\
[2000-2016] & &
\end{tabular}

Rates are per 100,000 and age adjusted to the 2000 US Standard Population (19 age groups, census P25-1130) standard. ENEC, esophageal neuroendocrine carcinoma; EACA, esophageal adenocarcinoma; ESqCC, esophageal squamous cell carcinoma. $\mathrm{P}<0.05$ was considered to be significant.

Table 2 TNM and SEER Staging for ENEC

\begin{tabular}{|c|c|c|}
\hline $\begin{array}{l}\text { ENEC (AJCC Stage } 7^{\text {th }} \\
\text { and SEER historic stage A) }\end{array}$ & Number & Percent (\%) \\
\hline \multicolumn{3}{|l|}{ T stage } \\
\hline $\mathrm{T} 1$ & 49 & 38.6 \\
\hline T2 & 12 & 9.4 \\
\hline T3 & 37 & 29.1 \\
\hline $\mathrm{T} 4$ & 29 & 22.8 \\
\hline \multicolumn{3}{|l|}{$\mathrm{N}$ stage } \\
\hline NO & 51 & 31.1 \\
\hline N1 & 98 & 59.8 \\
\hline N2 & 11 & 6.7 \\
\hline N3 & 4 & 2.4 \\
\hline \multicolumn{3}{|l|}{ M stage } \\
\hline MO & 101 & 51.0 \\
\hline M1 & 97 & 49.0 \\
\hline \multicolumn{3}{|l|}{ AJCC stage } \\
\hline I & 15 & 8.9 \\
\hline II & 28 & 16.6 \\
\hline III & 29 & 17.2 \\
\hline IV & 97 & 57.4 \\
\hline \multicolumn{3}{|l|}{ SEER stage } \\
\hline Localized & 95 & 14.8 \\
\hline Regional & 117 & 18.2 \\
\hline Distant & 351 & 54.5 \\
\hline Unstaged & 81 & 12.6 \\
\hline
\end{tabular}

$\mathrm{P}<0.004)$. Among different tumor grades, well differentiated tumor demonstrated a favorable effect [HR (95\% CI) by univariate analysis, $0.17(0.04-0.70), \mathrm{P}<0.014]$, while other grades did not have significant impact on patient survival. As for SEER histological stage, regional [HR (95\% CI): 1.44 (1.07-1.94), $\mathrm{P}=0.017$ ] and distant [HR (95\% CI): 2.99 (2.31-3.88), $\mathrm{P}<0.001]$ metastasis were detrimental, while unstaged tumors also showed unfavorable effect [HR (95\% CI): 2.27 (1.65-3.13), $\mathrm{P}<0.001]$. For AJCC stage, only stage IV exhibited negative effect on ENEC patient survival [HR (95\% CI) by univariate analysis: $3.87(1.99-7.55), \mathrm{P}<0.001]$.

As for CCS $(\mathrm{P}<0.001)$ and OS $(\mathrm{P}<0.001)$, ENEC demonstrated significantly worse prognosis compared with ESqCC and EACA (Figure 3). We then used survival analysis to evaluate the effectiveness of surgery, chemotherapy, and radiotherapy on ENEC. KaplanMeier analysis on both CSS and OS showed significant improvement after surgery (Figure $4 A, B$ ), radiotherapy (Figure $4 C, D$ ), and chemotherapy (Figure $4 E, F$ ) (all $\mathrm{P}<0.001)$. Moreover, we made a comparison of different treatment modalities, including surgery alone, chemotherapy alone, radiotherapy alone, and the combined therapy (surgery + chemotherapy; surgery + radiotherapy; chemotherapy + radiotherapy). Results revealed that surgery alone did not bring more survival benefit than radiotherapy alone or radiotherapy alone (Figure $5 A, B, C, D$ ). Meanwhile, chemotherapy alone was significantly better than radiotherapy alone on CSS (Figure 5E) $(\mathrm{P}=0.023)$. Moreover, compared with chemotherapy alone, surgery + chemotherapy can bring more significant benefits (both CSS and OS: $\mathrm{P}<0.001)$ (Figure $5 A, B)$. Similarly, compared with radiotherapy alone, surgery + radiotherapy have 

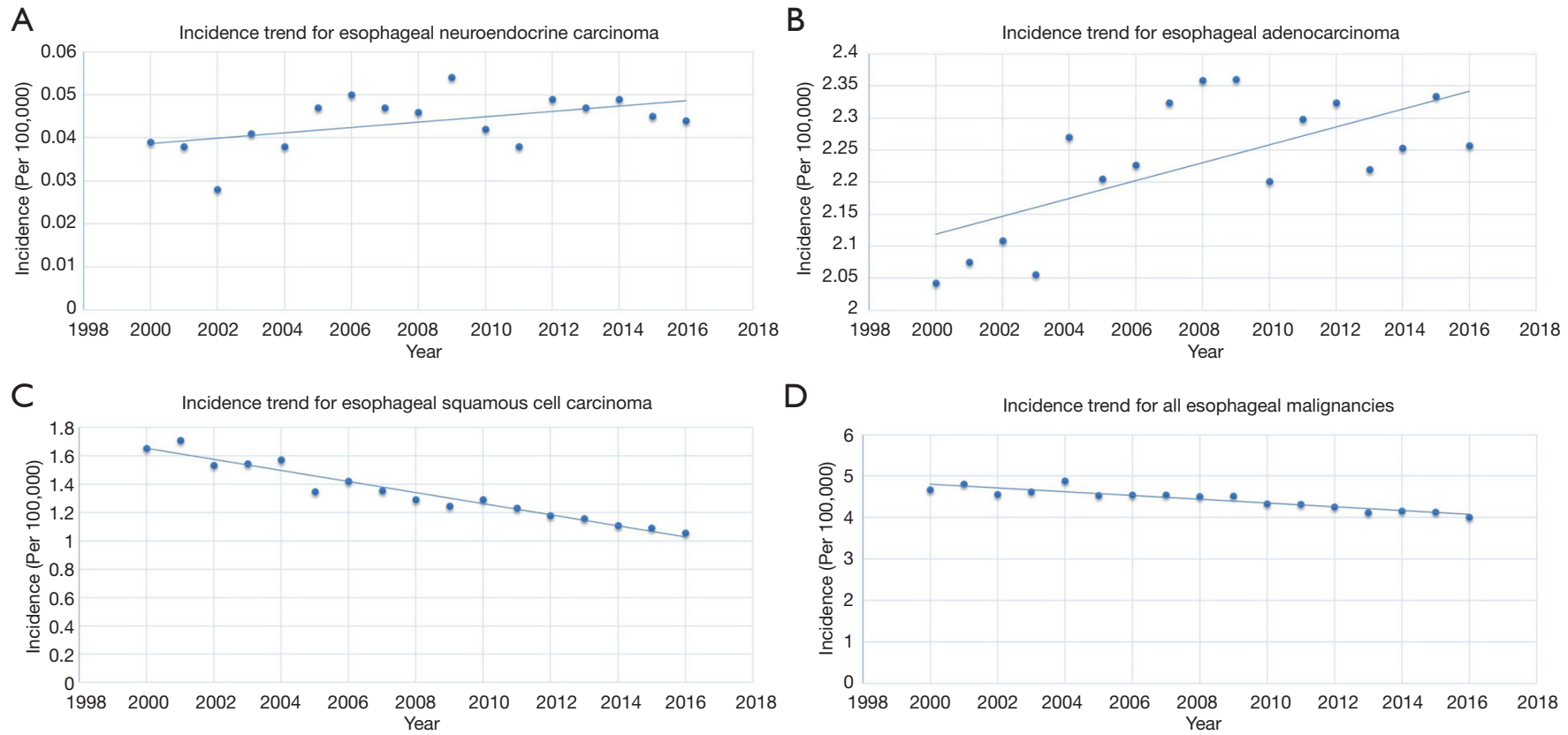

Figure 2 Incidence trend for (A) ENEC, (B) EACA, (C) ESqCC, and (D) all esophageal malignancies. Rates are per 100,000 and age adjusted to the 2000 US Standard Population (19 age groups, census P25 - 1130) standard. ENEC, esophageal neuroendocrine carcinoma; EACA, esophageal adenocarcinoma; ESqCC, esophageal squamous cell carcinoma.

more favorable effect on the survival of ENEC patients (CSS: $\mathrm{P}=0.024$; OS: $\mathrm{P}=0.010$ ) (Figure $5 C, D$ ). Besides, the combinational use of chemotherapy and radiotherapy was superior to chemotherapy alone (CSS and OS: $\mathrm{P}<0.001)$ and radiotherapy alone (CSS and OS: $\mathrm{P}<0.001)$ concerning the survival of ENEC patients (Figure $5 E, F$ ).

\section{Discussion}

As a subtype of ENENs, ENEC is extremely rare (1). Unlike well/intermediate-differentiated ENENs once referred to as carcinoid tumors, ENEC is poorly differentiated (12). The exact cause of ENEC stays unclear. Smoking and alcohol consumption are high-risk factors of ENEC (13). Cellular components in the gastrointestinal tract, like Merkel cells and Cajal cells, may be involved in the development of ENEC due to its observed association with other types of tumors in the digestive system. At the molecular level, positive stained indicators like p53, c-kit, and CK20 could participate in the initiation and progression of ENEC (14). There is a lack of population-based studies on ENEC. This study aimed to improve the understanding of ENEC in population regarding its incidence, treatment, and prognosis.
The term "neuroendocrine" refers to tumor cells with both "neuro" and "endocrine" properties. On the one hand, the "neuro" property is based on the finding of densecore granules. Such granules also appear in serotonergic neurons containing monoamines. On the other hand, the "endocrine" property suggests the synthesis and secretion of these monoamines. The neuroendocrine system ranges from endocrine glands (like pituitary and parathyroid), endocrine islet tissue (like pancreatic islets and thyroid) to scatter cells (like endocrine cells of digestive and respiratory tracts) in exocrine parenchyma. In most cases, primary tumors of NENs appear in the gastrointestinal tract (62$67 \%$ ) and lungs (22-27\%) (15-17). On the contrary, NENs seldom appear in the esophagus $(1,18)$. Therefore, ENEC is also rare, and in our study, the incidence of ENEC was 0.044 per 100,000 persons, much lower than those in EACA and ESqCC (Table 1 and Figure 2). Such phenomenon may be attributed to the underdevelopment of the neuroendocrine system in the esophagus.

In this study, despite the annual percentage change of ENEC was steady, we still observed the gradually increased number of patients with ENEC, which could be attributed to the improvement in its diagnosis. Clinical diagnosis of ENEC relies on examining pathological slices as well as 
Table 3 Parameters associated with overall survival in univariate and multivariate analysis for ENEC patients

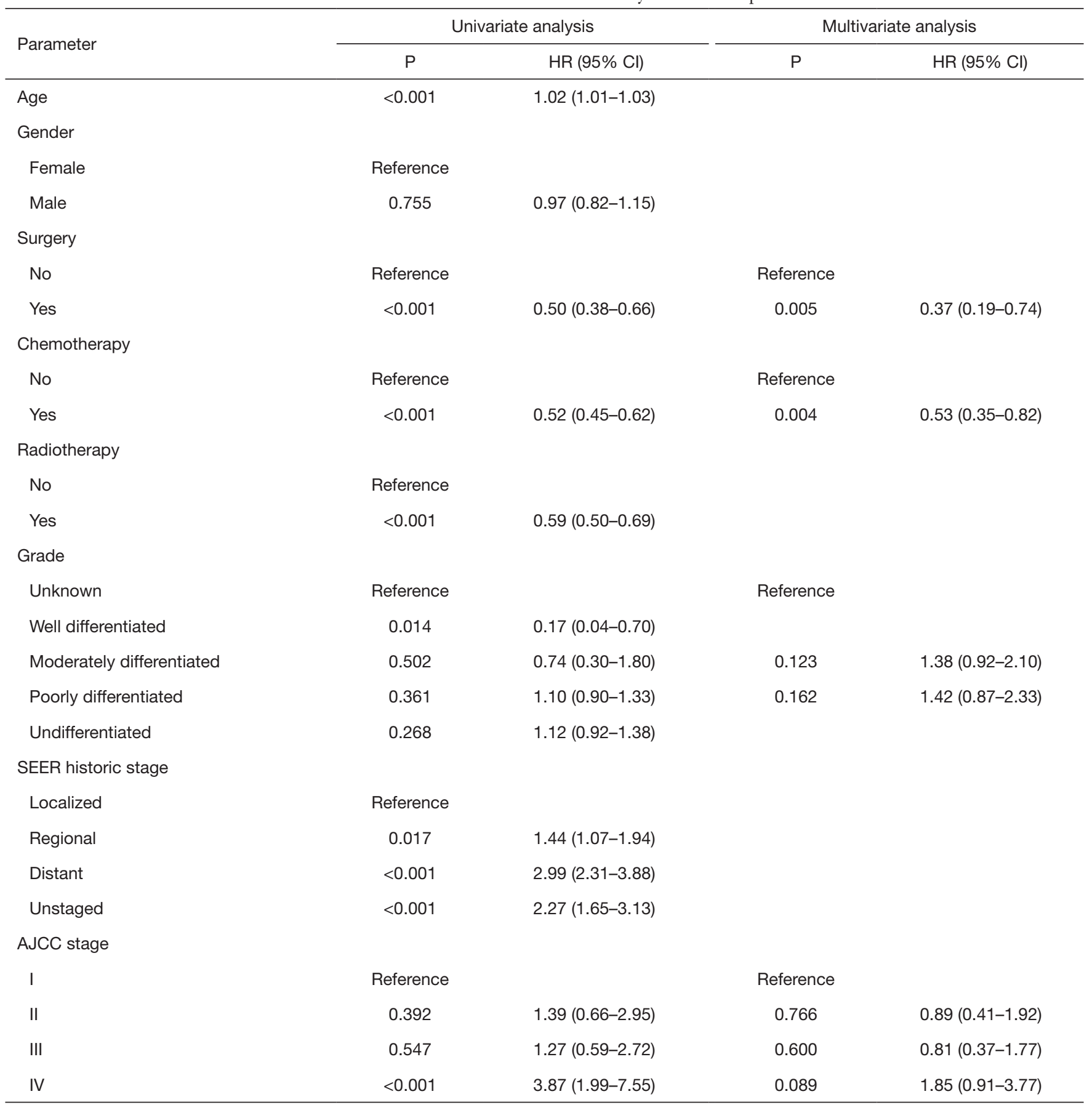

$\mathrm{P}<0.05$ was considered to be significant. OS, overall survival; ENEC, esophageal neuroendocrine carcinoma; AJCC, American Joint Committee on Cancer; HR, hazard ratio; $\mathrm{Cl}$, confidence interval. 

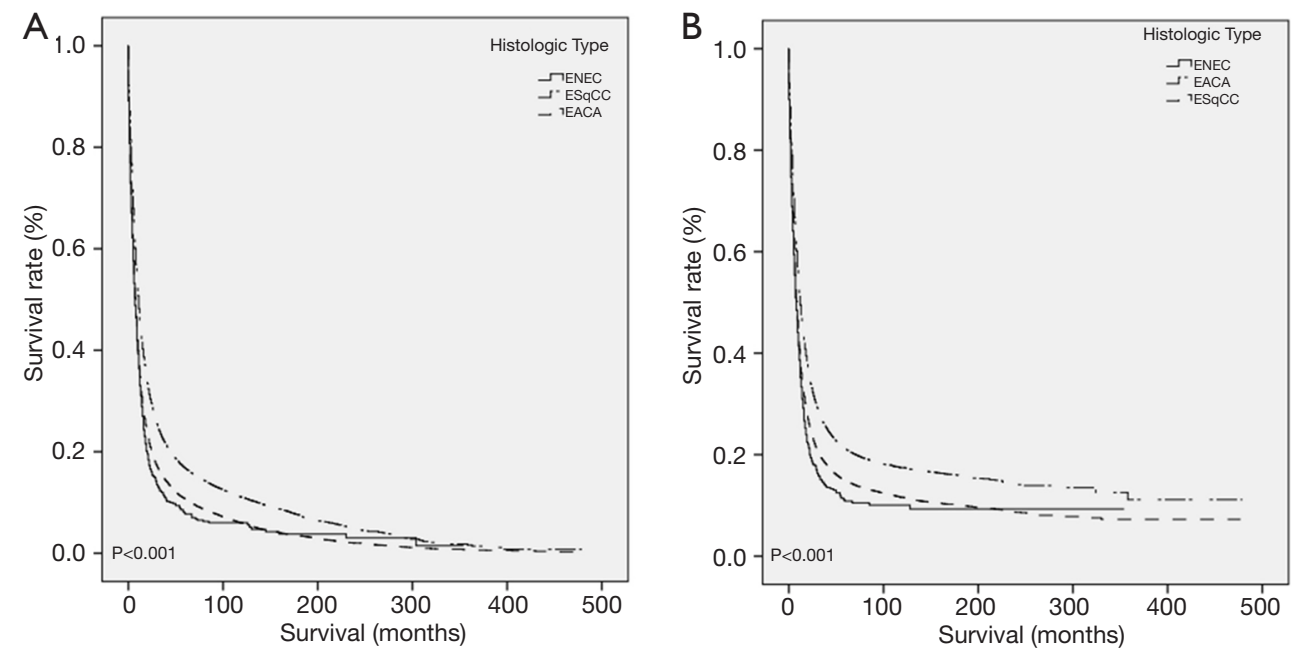

Figure 3 Survival analysis of ENEC, EACA, and ESqCC. (A) Cancer-specific survival (B) overall survival. ENEC, esophageal neuroendocrine carcinoma; EACA, esophageal adenocarcinoma; ESqCC, esophageal squamous cell carcinoma. $\mathrm{P}<0.05$ was considered to be significant.
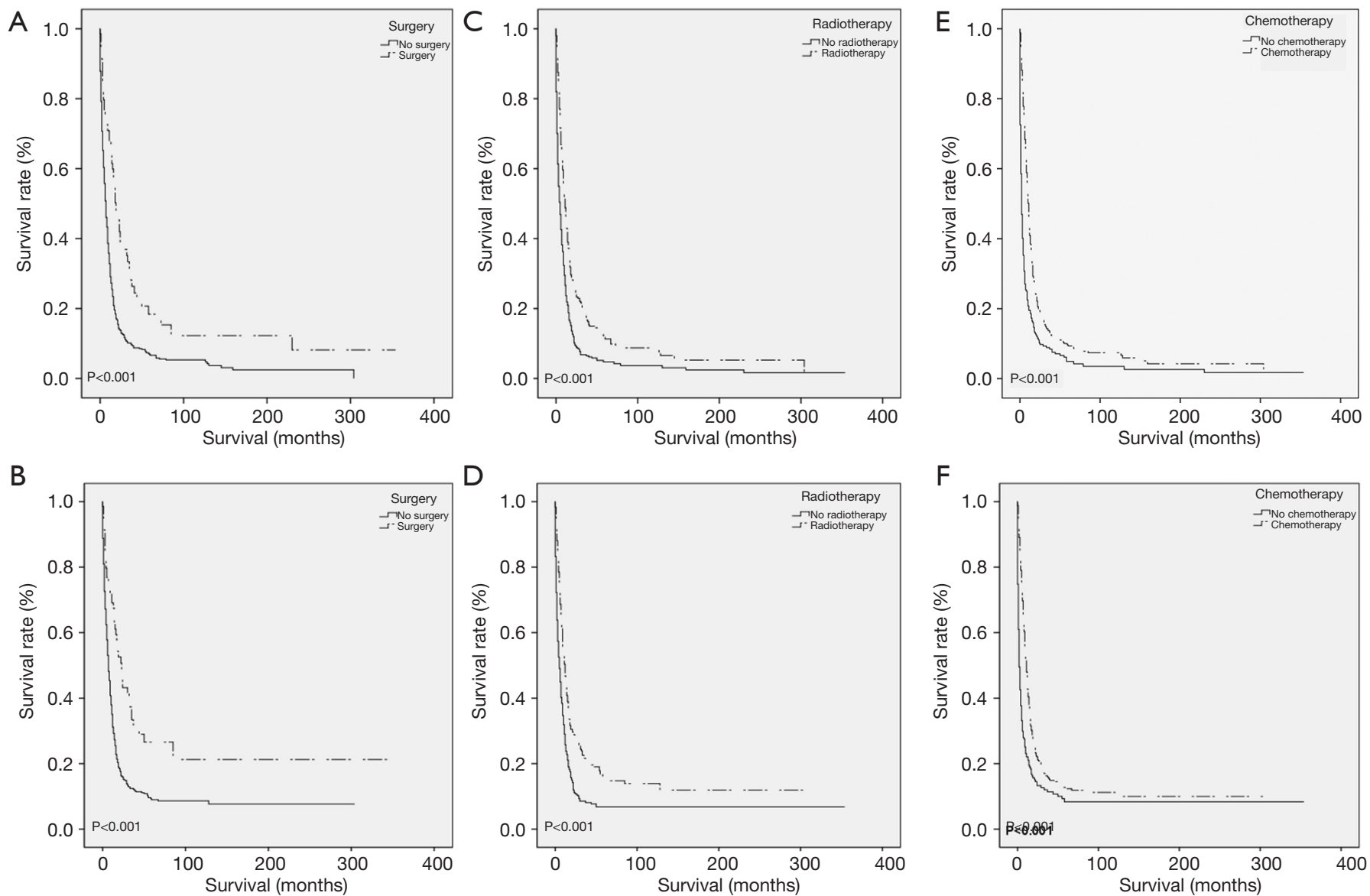

Figure 4 Effect of different treatments on the prognosis of patients with ENEC. CSS and OS of (A,B) surgery vs. no surgery, (C,D) radiotherapy $v s$. no radiotherapy, and (E,F) chemotherapy vs. no chemotherapy. ENEC, esophageal neuroendocrine carcinoma; CSS, cancerspecific survival; OS, overall survival. $\mathrm{P}<0.05$ was considered to be significant. 

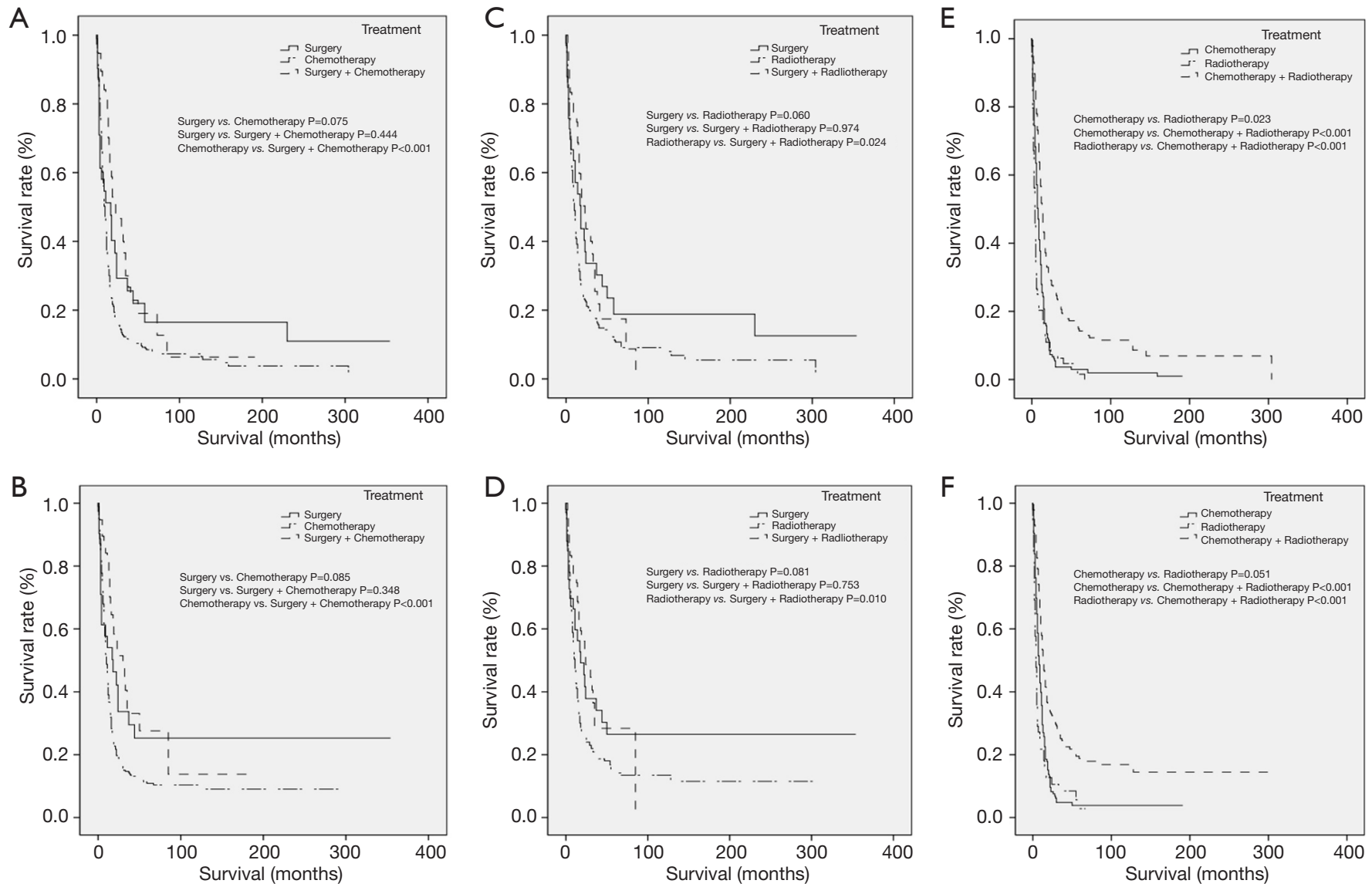

Figure 5 Survival analysis of ENEC patients adopting different therapies. CSS and OS of (A,B) surgery, chemotherapy, and surgery + chemotherapy, (C,D) surgery, radiotherapy, and surgery + radiotherapy, and (E,F) chemotherapy, radiotherapy, and chemotherapy + radiotherapy. ENEC, esophageal neuroendocrine carcinoma; CSS, cancer-specific survival; OS, overall survival. $\mathrm{P}<0.05$ was considered to be significant.

specific immunohistochemistry. Traditionally, ENEC is graded as G3 with a mitotic count of more than 20 per high power fields or a Ki-67 index of more than $20 \%$ (6). For ENEC, commonly used tumor markers also include CgA and 5-HIAA. Plasma CgA is correlated with tumor bulk, differentiation, and secretory activity, which, in turn, can predict treatment response and survival $(19,20)$. Besides, due to the "endocrine" property, tumor cells can also produce various bioactive amines and peptides, like serotonin, insulin, and somatostatin, leading to uncommon but unique clinical syndromes. Additionally, ENEC may be related to genetic mutations, such as mutations of multiple endocrine neoplasia type 1 and 2 (MEN-1 and MEN-2) (21-23). By analyzing the demographic features of our study cohort, we found that ENEC preferred old (mean: 67.5) and male (male vs. female: $67.6 \%$ vs. 32.4\%) patients. Besides, ENEC had a more significant proportion of high grades (I: $1.2 \%$; II: $0.9 \%$; III: $37.2 \%$; IV: 30.2\%) compared with EACA and $\mathrm{ESqCC}$ (Table 1). Therefore, as we expected, the prognosis of ENEC was worse than that of EACA and ESqCC (Figure 2), which also agreed with previous studies. As shown in Table 1, the proportion of patients who underwent surgery was considerably lower (surgery $v s$. no surgery: $10.2 \% v s .89 .8 \%$ ), compared with the proportion of patients adopting chemotherapy or radiotherapy. Such difference could be attributed to the aggressiveness of ENEC, which may cause metastasis at an early stage (9). Further analysis confirmed such speculation. Near half of the patients with ENEC were in the M1 stage, and more than half of patients with ENEC were at the AJCC IV stage, suggesting the loss of opportunities for surgical intervention. Besides, according to SEER staging analysis, only a small portion of 
patients with ENEC (14.8\%) was at a localized stage. All these results suggest that ENEC could be highly aggressive, and it is essential to deliver in-time treatments at an early stage.

Treatment strategies of ENEC have not been established due to its rarity. Current treatment regimens are generally based on the protocols of treating NECs on other sites like lungs (24). Surgery would be an ideal option when no metastasis occurs, while high rates of recurrence may raise a significant challenge to its efficacy (25). Whereas, a recent study still indicated that aggressive surgery could yield benefits for patient survival (26,27). Chemotherapy, such as irinotecan and platinum, is also among the firstline use. By now, multidisciplinary treatment, including adjuvant chemoradiotherapy, serves as an appropriate option, although ineffectiveness has been reported in largecell ENEC concerning clinical outcomes $(28,29)$. Besides, specific inhibition of molecules like CDK4/6 by using monoclonal antibody seems to be a promising strategy indicated by some cases (30).

Through univariate analysis, we found that surgery, chemotherapy, and radiotherapy can all benefit ENEC patient survival (Table 3). Multivariate analysis suggested surgery and chemotherapy could serve as independent protective prognostic factors for patients with ENEC. To date, the clinical rule of thumb is still to make surgical resection when possible. However, the role of surgery in ENEC remains controversial, despite our research and previous studies have demonstrated the survival benefit of surgical resection of ENEC (31,32). Meanwhile, different responses to surgery exist in patients with ENEC, indicating the need for further subgrouping patients with ENEC. Furthermore, compared with conventional esophagectomy, endoscopic submucosal dissection is less invasive. It can also achieve excellent results with early-stage tumors with a small diameter and a shallow depth (33).

Apart from surgery, chemotherapy and radiotherapy could play essential roles in ENEC treatments (9,34-37). In our finding, we also found chemotherapy was an independent prognostic factor for patients with ENEC (37). Moreover, previous studies showed that surgery combined with adjuvant therapy might be a good option for treating ENEC (9,38-41). Our findings also suggested more advantages of combinational therapy than monotherapy (Figure 5). Previously reported cases indicated that neoadjuvant chemotherapy might benefit patients with ENEC (38-41). However, the therapeutic effects of chemotherapy can be unsatisfactory due to low mitotic rate, high expression of the antiapoptotic protein $\mathrm{Bcl}-2$, and drug-resistant genes in poorly differentiated tumors (42). As for radiotherapy, somatostatin analogs like $117 \mathrm{Lu}$-octreotide could be used to increase survival rate, because of the high expression level of somatostatin receptor in tumor cells of ENEC. Somatostatin analogs can be biological therapeutics as well. By binding to their receptors, somatostatin analogs could inhibit the activity of adenylate cyclase and reduce calcium channel opening to block hormone synthesis and secretion $(43,44)$.

Admittedly, our research still has limitations, although we used population-based data. First, there was a lack of pathological slices and immunohistochemistry results, which we can directly observe in the SEER database. Second, there were insufficient details regarding surgery types, drug use, radiotherapy protocols, and comorbidities to support further analysis. Third, data input in different times and locations, or by different recorders, may introduce biases to some extent. Whereas, using the SEER database can yield significant benefits. The standardized database can also eliminate biases caused by geographical and institutional heterogeneity during analysis. Moreover, a considerable number of patients can help researchers to draw persuasive conclusions in analyzing rare malignancies like ENEC.

In summary, the incidence of ENEC is extremely low. Compared with EACA and ESqCC, ENEC had more aggressiveness and worse prognosis. While some clinical symptoms are similar to other esophageal cancers, it can have uncommon and unique manifestations due to its neuroendocrine properties. Pathological examinations are essential for diagnosis, while specific immunohistochemistry like Ki-67 is an important complement to pathological diagnosis. Surgery is still the mainstay in the treatment of ENEC, and adjuvant treatments, including chemotherapy and radiotherapy, can significantly improve patient prognosis.

\section{Conclusions}

Our population-based evidence showed that ENEC, as a rare cancer, had a worse prognosis compared with EACA and $\mathrm{ESqCC}$. Surgery, chemotherapy, and radiotherapy can all improve ENEC patient prognosis. Combinational therapy can yield a better prognosis than monotherapy.

\section{Acknowledgments}

We would like to extend our appreciation to the SEER 
database.

Funding: None.

\section{Footnote}

Reporting Checklist: The authors have completed the STROBE reporting checklist. Available at http://dx.doi. org/10.21037/tcr-19-2650

Conflicts of Interest: All authors have completed the ICMJE uniform disclosure form (available at http://dx. doi. org/10. 21037/tcr-19-2650). The authors have no conflicts of interest to declare.

Ethical Statement: The authors are accountable for all aspects of the work in ensuring that questions related to the accuracy or integrity of any part of the work are appropriately investigated and resolved. Due to omitted patient identifiers, this study did not have to get the approval of Institutional Review Board.

Open Access Statement: This is an Open Access article distributed in accordance with the Creative Commons Attribution-NonCommercial-NoDerivs 4.0 International License (CC BY-NC-ND 4.0), which permits the noncommercial replication and distribution of the article with the strict proviso that no changes or edits are made and the original work is properly cited (including links to both the formal publication through the relevant DOI and the license). See: https://creativecommons.org/licenses/by-nc-nd/4.0/.

\section{References}

1. Riihimaki $M$, Hemminki A, Sundquist $\mathrm{K}$, et al. The epidemiology of metastases in neuroendocrine tumors. Int J Cancer 2016;139:2679-86.

2. Park MI. Endoscopic treatment for early foregut neuroendocrine tumors. Clin Endosc 2013;46:450-5.

3. Yang L, Sun X, Zou Y, et al. Small cell type neuroendocrine carcinoma colliding with squamous cell carcinoma at esophagus. Int J Clin Exp Pathol 2014;7:1792-5.

4. Wilson CI, Summerall J, Willis I, et al. Esophageal collision tumor (Large cell neuroendocrine carcinoma and papillary carcinoma) arising in a Barrett esophagus. Arch Pathol Lab Med 2000;124:411-5.

5. Abe K, Yanagisawa D, Numakura S. Esophageal Neuroendocrine Carcinoma Presented as a Flat Depressed Lesion. Clin Gastroenterol Hepatol 2019;17:A31-A2.
6. Li QL, Zhang YQ, Chen WF, et al. Endoscopic submucosal dissection for foregut neuroendocrine tumors: an initial study. World J Gastroenterol 2012;18:5799-806.

7. Yang HX, Hou X, Liu QW, et al. Tumor location does not impact long-term survival in patients with operable thoracic esophageal squamous cell carcinoma in China. Ann Thorac Surg 2012;93:1861-6.

8. Ando T, Hosokawa A, Yamawaki H, et al. Esophageal small-cell carcinoma with syndrome of inappropriate secretion of antidiuretic hormone. Intern Med 2011;50:1099-103.

9. Deng HY, Ni PZ, Wang YC, et al. Neuroendocrine carcinoma of the esophagus: clinical characteristics and prognostic evaluation of 49 cases with surgical resection. J Thorac Dis 2016;8:1250-6.

10. Terada T. Small cell neuroendocrine carcinoma of the esophagus: report of 6 cases with immunohistochemical and molecular genetic analysis of KIT and PDGFRA. Int J Clin Exp Pathol 2013;6:485-91.

11. Lee CG, Lim YJ, Park SJ, et al. The clinical features and treatment modality of esophageal neuroendocrine tumors: a multicenter study in Korea. BMC Cancer 2014;14:569.

12. Hu Q, Jin P, Zhao X, et al. Esophageal neuroendocrine carcinoma complicated with unexpected hyperprocalcitonin: Case report and literature review. Medicine (Baltimore) 2018;97:e12219.

13. Ilett EE, Langer SW, Olsen IH, et al. Neuroendocrine Carcinomas of the Gastroenteropancreatic System: A Comprehensive Review. Diagnostics (Basel) 2015;5:119-76.

14. Egashira A, Morita M, Kumagai R, et al. Neuroendocrine carcinoma of the esophagus: Clinicopathological and immunohistochemical features of 14 cases. PLoS One 2017;12:e0173501.

15. Wolin EM. Advances in the Diagnosis and Management of Well-Differentiated and Intermediate-Differentiated Neuroendocrine Tumors of the Lung. Chest 2017;151:1141-6.

16. Hendifar AE, Marchevsky AM, Tuli R. Neuroendocrine Tumors of the Lung: Current Challenges and Advances in the Diagnosis and Management of Well-Differentiated Disease. J Thorac Oncol 2017;12:425-36.

17. Righi L, Gatti G, Volante M, et al. Lung neuroendocrine tumors: pathological characteristics. J Thorac Dis 2017;9:S1442-7.

18. Bohnenberger H, Dinter H, Konig A, et al. Neuroendocrine tumors of the thymus and mediastinum. J Thorac Dis 2017;9:S1448-57. 
19. Chou WC, Chen JS, Hung YS, et al. Plasma chromogranin A levels predict survival and tumor response in patients with advanced gastroenteropancreatic neuroendocrine tumors. Anticancer Res 2014;34:5661-9.

20. Seregni E, Ferrari L, Bajetta E, et al. Clinical significance of blood chromogranin A measurement in neuroendocrine tumours. Ann Oncol 2001;12 Suppl 2:S69-72.

21. Okubo Y, Yokose T, Motohashi O, et al. Duodenal Rare Neuroendocrine Tumor: Clinicopathological Characteristics of Patients with Gangliocytic Paraganglioma. Gastroenterol Res Pract 2016;2016:5257312.

22. Di Domenico A, Wiedmer T, Marinoni I, et al. Genetic and epigenetic drivers of neuroendocrine tumours (NET). Endocr Relat Cancer 2017;24:R315-34.

23. Derks JL, Leblay N, Lantuejoul S, et al. New Insights into the Molecular Characteristics of Pulmonary Carcinoids and Large Cell Neuroendocrine Carcinomas, and the Impact on Their Clinical Management. J Thorac Oncol 2018;13:752-66.

24. Giannetta E, Guarnotta V, Rota F, et al. A rare rarity: Neuroendocrine tumor of the esophagus. Crit Rev Oncol Hematol 2019;137:92-107.

25. Kikuchi Y, Shimada H, Yamaguchi K, et al. Systematic review of case reports of Japanese esophageal neuroendocrine cell carcinoma in the Japanese literature. Int Cancer Conf J 2019;8:47-57.

26. Nakao Y, Okino T, Yamashita YI, et al. Case report of aggressive treatments for large-cell neuroendocrine carcinoma of the esophagus. Int J Surg Case Rep 2019;60:291-5.

27. Tanaka Y, Hirata D, Fujii S, et al. Early-stage Neuroendocrine Carcinoma of the Esophagus Observed with Annual Endoscopy for Three Years. Intern Med 2019;58:1727-31.

28. Tomiyama T, Orino M, Nakamaru K, et al. Esophageal Large-Cell Neuroendocrine Carcinoma with Inconsistent Response to Treatment in the Primary and Metastatic Lesions. Case Rep Gastroenterol 2018;12:234-9.

29. Honma Y, Nagashima K, Hirano H, et al. Clinical outcomes of locally advanced esophageal neuroendocrine carcinoma treated with chemoradiotherapy. Cancer Med 2020;9:595-604.

30. Xu Y, Li X, Xu YF, et al. Poorly Differentiated Esophageal Neuroendocrine Carcinoma Treated With the CDK4/6 Inhibitor, Palbociclib: A Case Report and Literature Review. Am J Ther 2018;25:e595-8.

31. Jatoi A, Miller RC. Should we recommend surgery to patients with limited small cell carcinoma of the esophagus? J Thorac Oncol 2008;3:1373-6.

32. Muguruma K, Ohira M, Tanaka H, et al. Longterm survival of advanced small cell carcinoma of the esophagus after resection: a case report. Anticancer Res 2013;33:595-600.

33. Ishikawa-Kakiya Y, Nagami Y, Fujiwara Y. Intramucosal esophageal neuroendocrine carcinoma treated with endoscopic submucosal dissection and chemotherapy. Dig Endosc 2019;31:466.

34. Babu Kanakasetty G, Dasappa L, Lakshmaiah KC, et al. Clinicopathological Profile of Pure Neuroendocrine Neoplasms of the Esophagus: A South Indian Center Experience. J Oncol 2016;2016:2402417.

35. Patta A, Fakih M. First-line cisplatin plus etoposide in high-grade metastatic neuroendocrine tumors of colon and rectum (MCRC NET): review of 8 cases. Anticancer Res 2011;31:975-8.

36. Chen WW, Wang F, Chen S, et al. Detailed analysis of prognostic factors in primary esophageal small cell carcinoma. Ann Thorac Surg 2014;97:1975-81.

37. Chen SB, Yang JS, Yang WP, et al. Treatment and prognosis of limited disease primary small cell carcinoma of esophagus. Dis Esophagus 2011;24:114-9.

38. Xu L, Li Y, Liu X, et al. Treatment Strategies and Prognostic Factors of Limited-Stage Primary Small Cell Carcinoma of the Esophagus. J Thorac Oncol 2017;12:1834-44.

39. Sarkaria IS, Iyoda A, Roh MS, et al. Neoadjuvant and adjuvant chemotherapy in resected pulmonary large cell neuroendocrine carcinomas: a single institution experience. Ann Thorac Surg 2011;92:1180-6; discussion 6-7.

40. Yamamoto M, Ozawa S, Koyanagi K, et al. Effectiveness of neoadjuvant chemotherapy with etoposide and cisplatin followed by surgery for esophageal neuroendocrine carcinoma: a case report. J Thorac Dis 2018;10:E450-5.

41. Akiyama Y, Iwaya T, Shioi Y, et al. Effectiveness of neoadjuvant chemotherapy with cisplatin and irinotecan followed by surgery on small-cell carcinoma of the esophagus: A case report. Int J Surg Case Rep 2015;17:121-5.

42. Yazici O, Ozdemir NY, Sendur MA, et al. Current approaches for prophylactic cranial irradiation in extrapulmonary small cell carcinoma. Curr Med Res Opin 2014;30:1327-36.

43. Carmona-Bayonas A, Jimenez-Fonseca P, Lamarca A, et al. Prediction of Progression-Free Survival in Patients With Advanced, Well-Differentiated, Neuroendocrine Tumors 
Being Treated With a Somatostatin Analog: The GETNETRASGU Study. J Clin Oncol 2019:JCO1900980.

44. Boudreaux JP, Klimstra DS, Hassan MM, et al. The NANETS consensus guideline for the diagnosis and

Cite this article as: $\mathrm{Li} \mathrm{Z,} \mathrm{Hu} \mathrm{J,} \mathrm{Chen} \mathrm{P,} \mathrm{Zeng} \mathrm{Z.} \mathrm{Incidence,}$ treatment, and survival analysis in esophageal neuroendocrine carcinoma population. Transl Cancer Res 2020;9(7):4317-4329. doi: 10.21037/tcr-19-2650 management of neuroendocrine tumors: well-differentiated neuroendocrine tumors of the Jejunum, Ileum, Appendix, and Cecum. Pancreas 2010;39:753-66. 